\title{
Orthologs of Human Disease Associated Genes and RNAi Analysis of Silencing Insulin Receptor Gene in Bombyx mori
}

\author{
Zan Zhang ${ }^{1}$, Xiaolu Teng ${ }^{1}$, Maohua Chen ${ }^{2}$ and Fei Li ${ }^{1, *}$ \\ 1 Department of Entomology, College of Plant Protection, Nanjing Agricultural University, \\ Nanjing 210095, China; E-Mails: zhangzan125@gmail.com (Z.Z.); \\ tengxiaolu177546@gmail.com (X.T.) \\ 2 College of Plant Protection, Northwest A\&F University, Taicheng Road, Yangling 712100, \\ Shaanxi, China; E-Mail: cmhwh1@hotmail.com \\ * Author to whom correspondence should be addressed; E-Mail: lifei@njau.edu.cn; \\ Tel.: +86-25-8439-9025; Fax: +86-25-8439-9920.
}

External Editor: Bing Yan

Received: 1 June 2014; in revised form: 24 August 2014 / Accepted: 19 September 2014 / Published: 9 October 2014

\begin{abstract}
The silkworm, Bombyx mori L., is an important economic insect that has been domesticated for thousands of years to produce silk. It is our great interest to investigate the possibility of developing the B. mori as human disease model. We searched the orthologs of human disease associated genes in the $B$. mori by bi-directional best hits of BLAST and confirmed by searching the OrthoDB. In total, 5006 genes corresponding to 1612 kinds of human diseases had orthologs in the B. mori, among which, there are 25 genes associated with diabetes mellitus. Of these, we selected the insulin receptor gene of the $B$. mori $(B m-I N S R)$ to study its expression in different tissues and at different developmental stages and tissues. Quantitative PCR showed that Bm-INSR was highly expressed in the Malpighian tubules but expressed at low levels in the testis. It was highly expressed in the 3rd and 4th instar larvae, and adult. We knocked down Bm-INSR expression using RNA interference. The abundance of Bm-INSR transcripts were dramatically reduced to $\sim 4 \%$ of the control level at 6 days after dsRNA injection and the RNAi-treated B. mori individuals showed apparent growth inhibition and malformation such as abnormal body color in black, which is the typical symptom of diabetic patients. Our results demonstrate that B. mori has potential use as an animal model for diabetic mellitus research.
\end{abstract}


Keywords: Bombyx mori; human diseases model; diabetes mellitus; INSR; dsRNA

\section{Introduction}

Animal models are a helpful for studying molecular mechanisms of human disease as well as for drug testing. The most widely used animal models are mouse, Mus musculus and Rat, Rattus rattus, which have been used to model many human diseases, including neurological and behavior disorders [1-4]. The advantages of using rodents as disease models are apparent. These mammals are evolutionally close to humans and share similar gene regulation mechanisms. Transgenic mice have become an efficient method to produce a disease model [5]. Besides mouse, the dog was used to model human non-Hodgkins lymphoma [6]. However, mouse, rat and dog have a long life cycle and the costs are high. To overcome these disadvantages, the fruitfly, Drosophila melanogaster, was widely used for modeling lots of human disorders such as neurodegenerative diseases [7-9], Huntington's disease[10], Barth syndrome [10,11], Parkinson's disease [12], Alzheimer's disease [13], cancer [14], heart disease [15-17], and Multiple endocrine neoplasia [18]. The success of using insect models for human diseases is exciting and highly useful since it provides a cost-effective strategy in disease research and drug screening.

However, it is impossible to use one animal to model all kinds of human disorders and choosing the right animal model is important. The choice often considers the nature of the disease and characteristics of the animal [19-21]. The B. mori genome was published in 2004 and many B. mori genes have been well studied [22,23]. The advantages of using the silkworm to model human diseases are apparent: (1) The life cycle of silkworm is about one month, which is much shorter than mice and rats. The shorter life cycle will accelerate the research; (2) Since the B. mori has been domesticated for more than two thousand years, it is very easy to be maintained in the lab with artificial diets; and (3) Transgenic B. mori is easy to be obtained with routine methods [24-26]. The silkworm has been widely used as the bioreactor to express human genes for producing drugs or vaccines [27-29]. Because of these advantages, the $B$. mori has been successfully used to model human disorders such as Parkinson's disease [30], and human sepiapterin reductase deficiency [31]. The B. mori was also helpful in screening drugs. It was successfully used to screen ant-diabetic drugs by feeding a high glucose-containing diet [32]. Five proteins were induced by a high-glucose diet in the silkworm [33]. Bombyxin is an insect insulin-related peptide. Bombyxin can reduce the concentration of the major hemolymph sugar trehalose in larvae [34,35]. Glucose can stimulates the release of Bombyxin in B. mori [36]. These studies suggest that it is possible to use the $B$. mori to model insulin-related human diseases such as diabetes. Here, we searched the orthologs of human disease-associated genes in the B. mori, and then selected the insulin receptor gene in B. mori (Bm-INSR) for further gene expression and RNAi analysis.

\section{Results and Discussion}

\subsection{Orthologs of Diseased-Associated Genes in the B. mori}

We obtained 69,040 protein sequences corresponding to 2629 kinds of human disorders from the database of Online Mendelian Inheritance in Man (OMIM) [37]. The 14,623 B. mori genes 
were downloaded from the SilkDB (v2.0). These genes contain intact open reading frames (ORF). We identified orthologs by using bidirectional best hits, which is a widely used method. The $E$-value cutoff of Blast is 0.00001. As a result, 5006 B. mori genes were orthologs of human disease-associated genes, corresponding to 1612 kinds of human disorders. We classified human diseases into 18 categories, showing that silkworm (B. mori) orthologs of human disease-associated (SOHD) genes are mainly related with skeletal disease, head and neck disease, neurologic disease, and growth diseases (Figure 1, Table S1) [38]. In these SOHD genes, 4941 B. mori genes have orthologs in D. melanogaster, supporting the utility of B. mori as an alternative model (Table S1).

Figure 1. The numbers of the silkworm orthologs in different categories of human diseases.

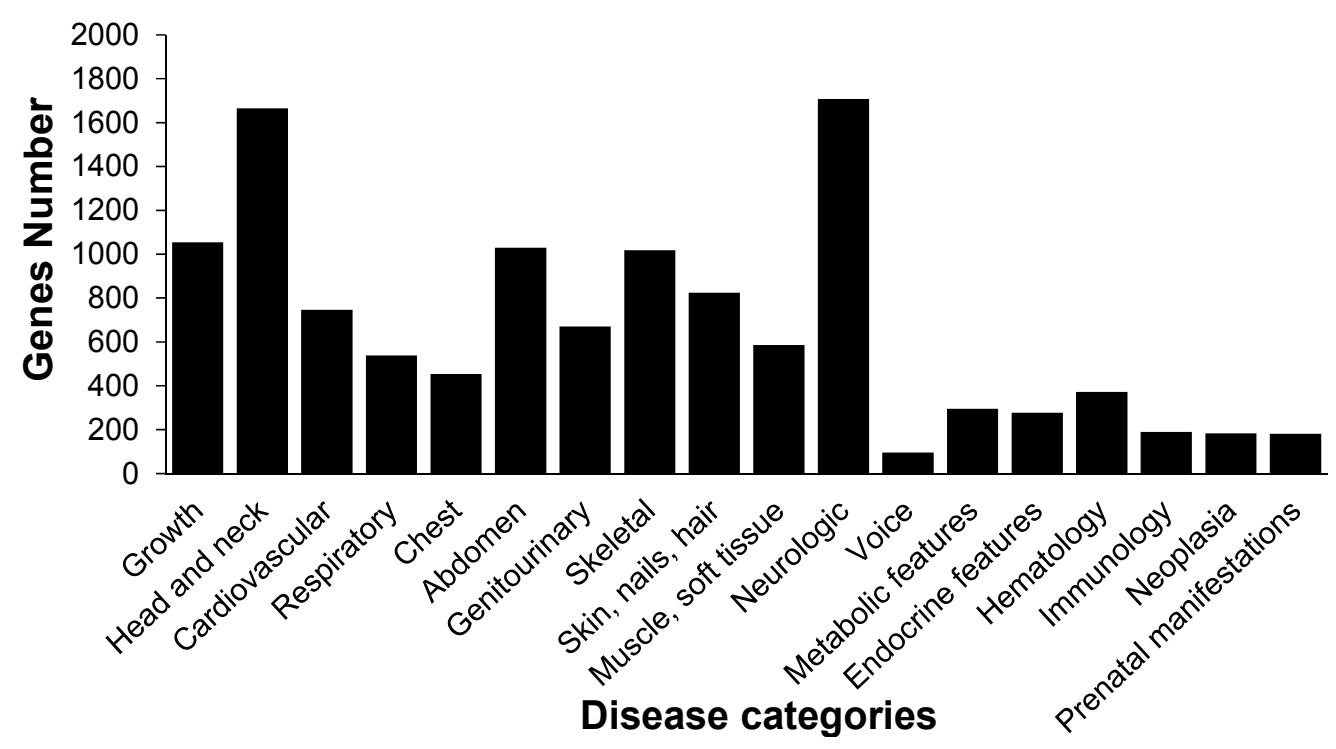

Figure 2. Gene ontology (GO) term classifications of silkworm orthologs of human disease. $\mathrm{GO}$ analysis was performed using Blast2go. The GO terms were classified into different categories at level 2.

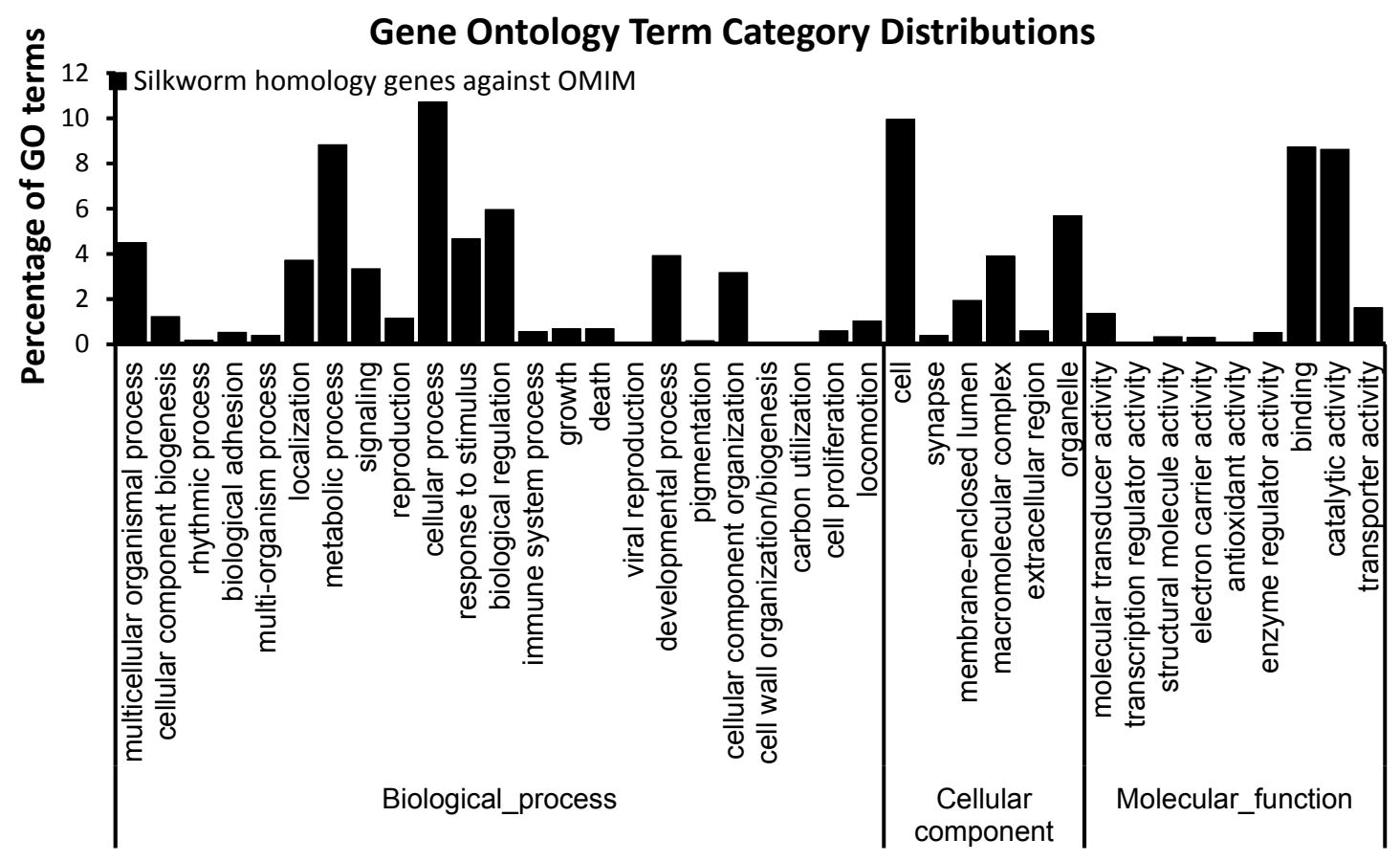


Gene ontology analysis showed that SOHD genes were rich in "symplast" at the cellular component system but lacked "protein tag" and "nutrient reservoir activity" at the molecular function system (Figure 2). Pathway analysis indicated that SOHD genes were rich in carbohydrate metabolism and lipid metabolism. For organismal system, SOHD genes were rich in digestive system, immune system and nervous system. For human disease system, SOHD genes were rich in infectious, cardiovascular and cancer (Figure 3).

Figure 3. Pathway analysis of silkworm genes associated with human disease. The analysis was performed using BLASTP against KEGG database. The pathways were clustered with three different systems, metabolism system, organismal system and human disease.

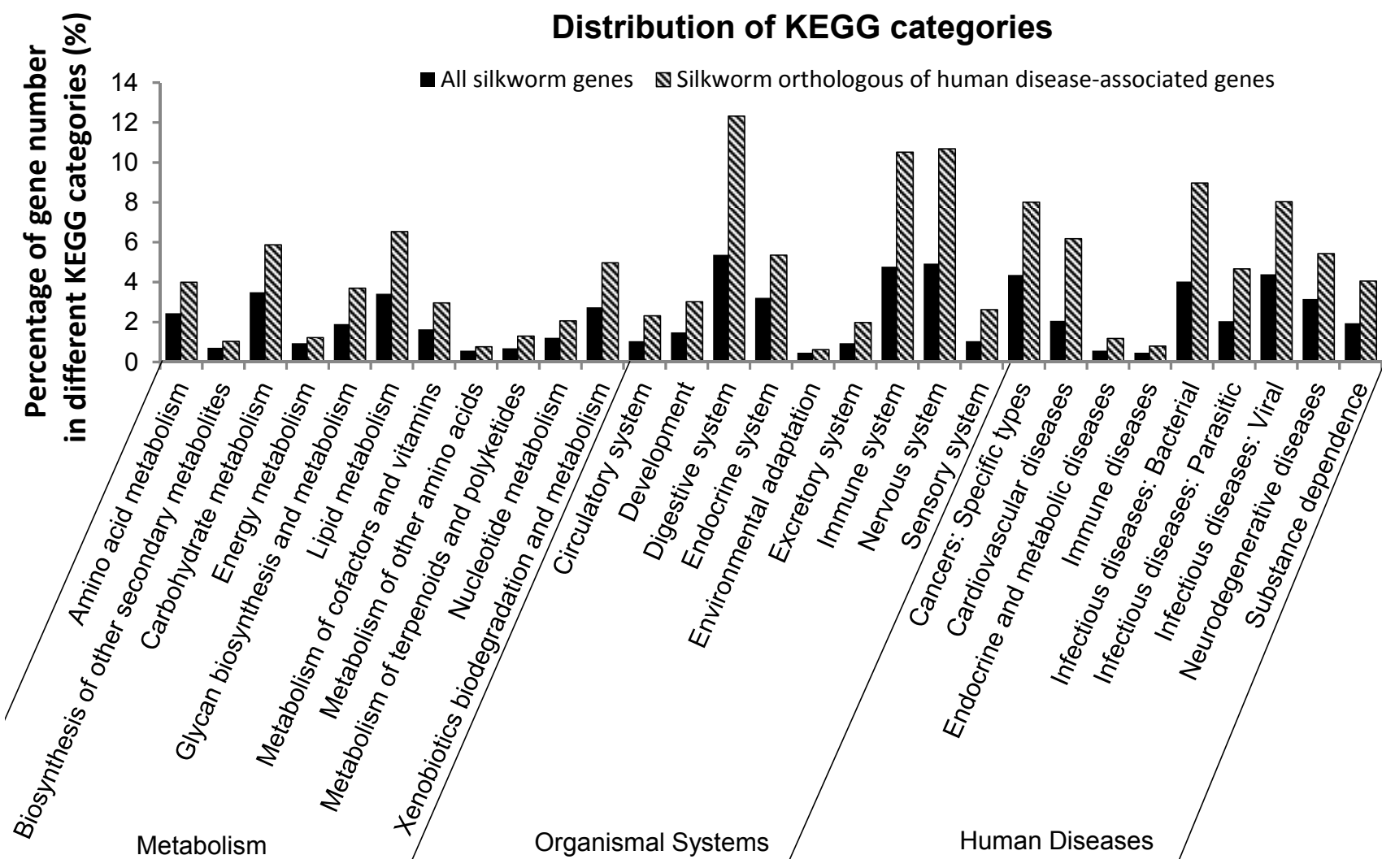

\subsection{Insulin Receptor Genes in the B. mori}

We found that $25 \mathrm{~B}$. mori genes were orthologs of human genes associated with diabetes mellitus (Table 1). Insulin receptor-like protein precursor in the B. mori (Bm-INSR, SilkDB ID: BGIBMGA003654) shares 56\% sequence similarity with human insulin (GenBank ID: EAW69046). Domain analysis showed that INSR genes in human, mouse and B. mori were highly conserved. They all contained two receptor L domains (Receptor L), furin-like repeats (cysteine-rich region), fibronectin type 3 domain (FN3) and protein kinases catalytic domain (PTKc) [39,40], indicated that they had the similar function. Thus, we selected the Bm-INSR for further analysis. 
Table 1. The silkworm orthologs of human genes associated with diabetes mellitus.

\begin{tabular}{|c|c|c|c|}
\hline $\begin{array}{l}\text { Accession Numbers } \\
\text { of Human Genes }\end{array}$ & SilkDB ID & $E$-Value & Diabetes Disorders \\
\hline NP_005318.3 & BGIBMGA007360 & $2.00 \times 10^{-89}$ & Hyperinsulinemic hypoglycemia, familial, 4, 609975. \\
\hline NP_619726.2 & BGIBMGA006839 & $5.00 \times 10^{-28}$ & $\begin{array}{l}\text { Insulin resistance, severe, digenic, } 604367 . \\
\text { Obesity, resistance to Diabetes, type 2, } 125853 \text {. }\end{array}$ \\
\hline NP_001073285 & BGIBMGA003800 & $2.00 \times 10^{-64}$ & $\begin{array}{l}\text { Diabetes mellitus, insulin-resistant, with acanthosis } \\
\text { nigricans, } 610549 . \\
\text { Hyperinsulinemic hypoglycemia, familial, 5, } 609968 .\end{array}$ \\
\hline BAC11220.1 & BGIBMGA009492 & $4.00 \times 10^{-22}$ & Diabetes mellitus, noninsulin-dependent $1,601283$. \\
\hline NP_000343.2 & BGIBMGA006882 & $9.00 \times 10^{-50}$ & $\begin{array}{l}\text { Diabetes mellitus, noninsulin-dependent, } 125853 . \\
\text { Diabetes mellitus, permanent neonatal, } 606176 . \\
\text { Diabetes mellitus, transient neonatal 2, } 610374 . \\
\text { Hyperinsulinemic hypoglycemia, familial, 1, } 256450 .\end{array}$ \\
\hline NP_005996.2 & BGIBMGA002273 & $1.00 \times 10^{-35}$ & \multirow{9}{*}{$\begin{array}{l}\text { Diabetes mellitus, noninsulin-dependent, susceptibility } \\
\text { to, } 125853 \text {. }\end{array}$} \\
\hline BAA91102.1 & BGIBMGA007364 & $1.00 \times 10^{-25}$ & \\
\hline NP_001007226 & BGIBMGA004315 & $1.00 \times 10^{-8}$ & \\
\hline NP_006539.3 & BGIBMGA007473 & $1.00 \times 10^{-7}$ & \\
\hline NP_060244.2 & BGIBMGA011966 & 0 & \\
\hline NP_776250.2 & BGIBMGA005779 & $1.00 \times 10^{-72}$ & \\
\hline NP_002818.1 & BGIBMGA002096 & $1.00 \times 10^{-66}$ & \\
\hline AAA52569.1 & BGIBMGA010881 & $1.00 \times 10^{-8}$ & \\
\hline NP_001033.1 & BGIBMGA002023 & $3.00 \times 10^{-93}$ & \\
\hline NP_003042.3 & BGIBMGA003138 & $6.00 \times 10^{-40}$ & Hyperinsulinemic hypoglycemia, familial, 7, 610021. \\
\hline BAF83535.1 & BGIBMGA003740 & $6.00 \times 10^{-44}$ & \multirow{3}{*}{ Diabetes mellitus, noninsulin-dependent. } \\
\hline BAH12783.1 & BGIBMGA004629 & $3.00 \times 10^{-12}$ & \\
\hline NP_000331.1 & BGIBMGA010740 & $3.00 \times 10^{-51}$ & \\
\hline NP_005262.1 & BGIBMGA014352 & $1.00 \times 10^{-38}$ & Hyperinsulinism-hyperammonemia syndrome, 606762. \\
\hline NP_002702.2 & BGIBMGA011660 & $3.00 \times 10^{-19}$ & Insulin resistance, severe, digenic, 604367. \\
\hline EAX02222.1 & BGIBMGA008597 & $3.00 \times 10^{-22}$ & \multirow{2}{*}{ Insulin-like growth factor I, resistance to, 270450.} \\
\hline NP_000866.1 & BGIBMGA008240 & $3.00 \times 10^{-19}$ & \\
\hline AAA93480.1 & BGIBMGA003134 & $4.00 \times 10^{-10}$ & Maturity-onset diabetes of the young 6, 606394 . \\
\hline NP_002491.2 & BGIBMGA008390 & $2.00 \times 10^{-9}$ & Diabetes mellitus, noninsulin-dependent, 125853. \\
\hline
\end{tabular}

\subsection{The Expression of Insulin Receptor Gene in the B. mori}

We investigated the expressions of Bm-INSR gene in six tissues including head, Malpighian tubules, ovary, testis and silk gland. The housekeeping gene $R P 49$ was used as the reference gene in qPCR. $B m-I N S R$ was highly expressed in the Malpighian tubule but expressed at low levels in the testis (Figure 4A). The Malpighian tubule is a type of excretory and osmoregulatory system. Though it does not have a digestive function, the Malpighian tubule can release the waste of digested food. It has been reported that an $\mathrm{H}^{+}$-dependent trehalose transporter can induce the reabsorption of trehalose in Malpighian tubules [41]. This can partially explains the reason of high expression of Bm-INSR gene in the Malpighian tubule. 
The egg, larvae at the first day of each instar (1L1d, 2L1d, 3L1d, 4L1d 5L1d), pupa and adult B. mori were also chosen for analysis. The results showed that Bm-INSR gene was highly expressed in the 3rd and the 4th instar larvae, followed by egg and adult. It was expressed at low levels in the 2nd and the 5th instar larvae (Figure 4B). The expression peaks of insulin receptor gene in the 3rd and 4th instars indicated that insulin receptor gene is related with food metabolism because the $B$. mori larvae eats lots of mulberry leaves at these two stages. It has been reported that glucose in the food stimulates the release of Bombyxin in B. mori [36].

Figure 4. Relative abundance of Bm-INSR gene in different tissues and developmental stages. (A) different tissues; and (B) different developmental stages. 1L1D, 2L1D, 3L1D, 4L1D, 5L1D: First day of each instar (Tukey test, $p<0.05$. "a" and "b", same letters in figure differentiated no significantly, different letters differentiated significantly). The gene expression levels were examined using quantitative real time PCR. The gene RP49 was used as the internal control.
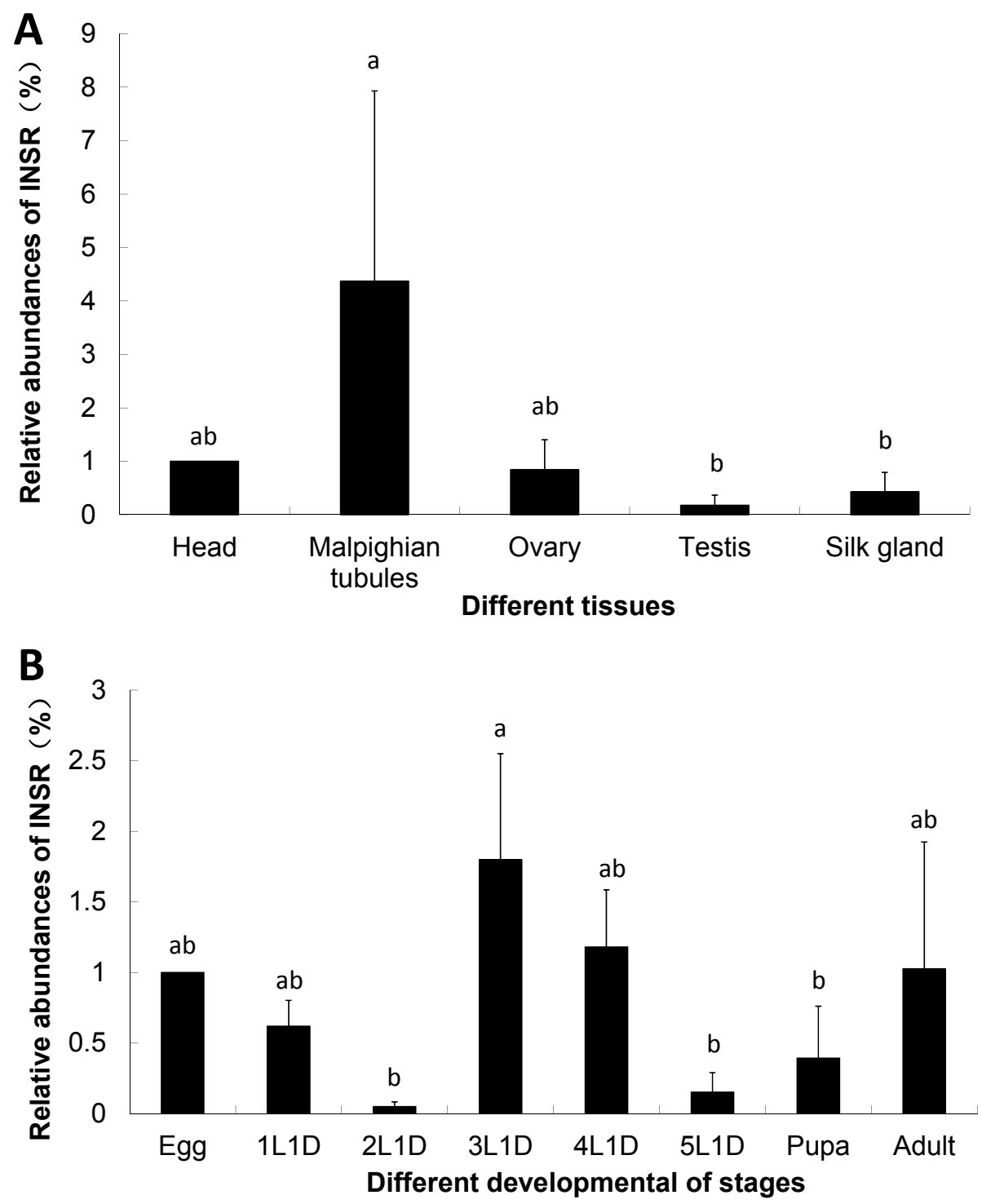

The expression of the Bm-INSR was higher in the Malpighian tubules than in testis and silk gland (Figure 4A). Since the Malpighian tubule is a type of excretory and osmoregulatory system, which 
can release the food waste, the reabsorption of trehalose was reported in Malpighian tubules. The high expression of Bm-INSR gene in this tissue indicated that the Bm-INSR is associated with food metabolism.

\subsection{Knockdown of the Insulin Receptor Genes in the B. mori}

DsRNA was designed based on Bm-INSR gene sequence and then was synthesized using T7 RiboMAX kit (Promega, Madison, WI, USA). One $\mu \mathrm{L}$ of dsRNA $\left(5000 \mathrm{ng} \cdot \mu \mathrm{L}^{-1}\right)$ was injected into the 3rd instar larvae. The dsRNA designed from GFP gene sequence was used as the negative control. To examine RNAi efficiency, we collected RNAi-treated larvae at 48, 96, and $124 \mathrm{~h}$ after dsRNA-injection. Real time PCR analysis showed that the abundance of Bm-INSR gene was decreased to $50.2 \%$ of the control level at $96 \mathrm{~h}$ post-injection (Figure 5). The mRNA abundance of the Bm-INSR gene dramatically decreased to $4 \%$ at $124 \mathrm{~h}$ post-injection $(p<0.05)$. The results showed that we silenced the Bm-INSR successfully.

Figure 5. Relative abundance of Bm-INSR genes after dsRNA injection. Ds-GFP signifies the negative control groups. ds-INSR represents dsRNA treated groups. Asterisks indicate statistically significant difference $(t$-test, $p<0.01)$. The gene expressions were examined using quantitative real time PCR. The gene RP49 was used as the internal control.

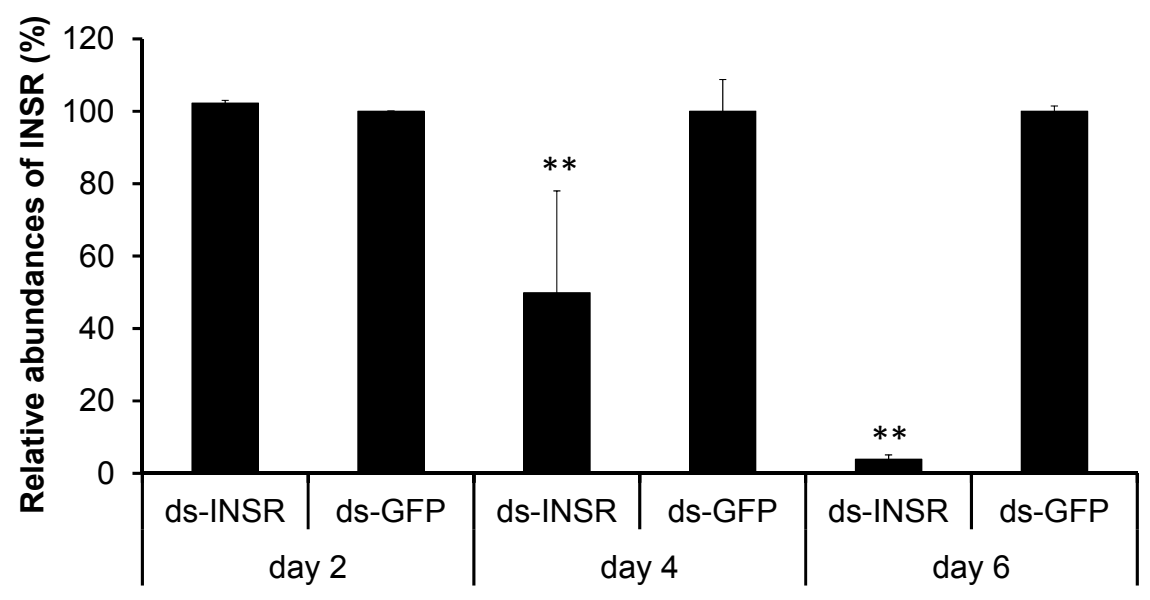

\subsection{The Impact of INSR Gene Knockdown on B. mori Development}

We chose ten RNAi-treated B. mori individuals to measure their weights and lengths. At seven days after dsRNA injection, the average body weight of RNAi-treated groups is $0.879 \pm 0.021 \mathrm{~g}$, which was less than that of the control group $(1.00 \pm 0.35 \mathrm{~g})$. The significant difference was observed between the third and sixth days post-injection ( $p<0.01, t$-test, Figure 6A). However, knockdown of the Bm-INSR gene did not affect body length. At seven days post-injection, the average length of the RNAi-treated group was $4.27 \pm 0.57 \mathrm{~cm}$, similar to the control group $(4.4 \pm 0.46 \mathrm{~cm})$ (Figure 6B). In the RNAi-treated group, $20 \%$ of individuals showed growth retardation. About $20 \%$ of RNAi-treated B. mori became black in body color (Figure 7). The mRNA levels of Bm-INSR gene were significantly decreased in the silkworm that became black in body color, which might be associated with melanin synthesis, because there was not any silkworm that became black in the control group. So, it is unlikely that RNAi induces the body color change. It has been reported that abnormal pigmentation is typically a sign of an innate immune response and insulin signaling is closely connected with immunity [41-43]. 
The results indicated that dsRNA-treated silkworms showed two typical symptoms of diabetes patients. One was weight reduction, and the other was black body color. These results demonstrated that Bm-INSR has important functions in silkworm development and B. mori could be used as an animal model for diabetes mellitus by RNA interference.

Figure 6. The change of the body length and weight in larvae of different treatments after injection. Ten animals were used in each experiment. Ds-GFP: Group after injection the dsRNA used to silence GFP. ds-INSR: Group after injection the dsRNA used to silence INSR. 1D, 2D, 3D, 4D, 5D, 6D, 7D: One days, two days ,three days ,four days, five days, six days and seven days after injection. (A) shows the change of weight of the insect body in 7 days and (B) shows the change of length of the insect body in 7 days.
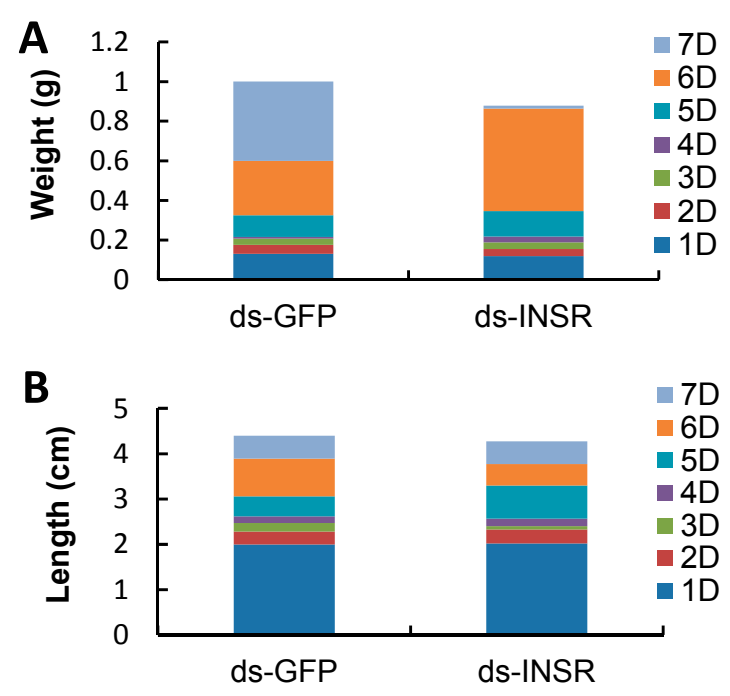

Figure 7. Growth inhibition of dsRNA-treated larvae. (A) The growth retardation compared with the control at 10 days after injection; and (B) The RNAi-treated silkworm in black body color at 10 days after injection.

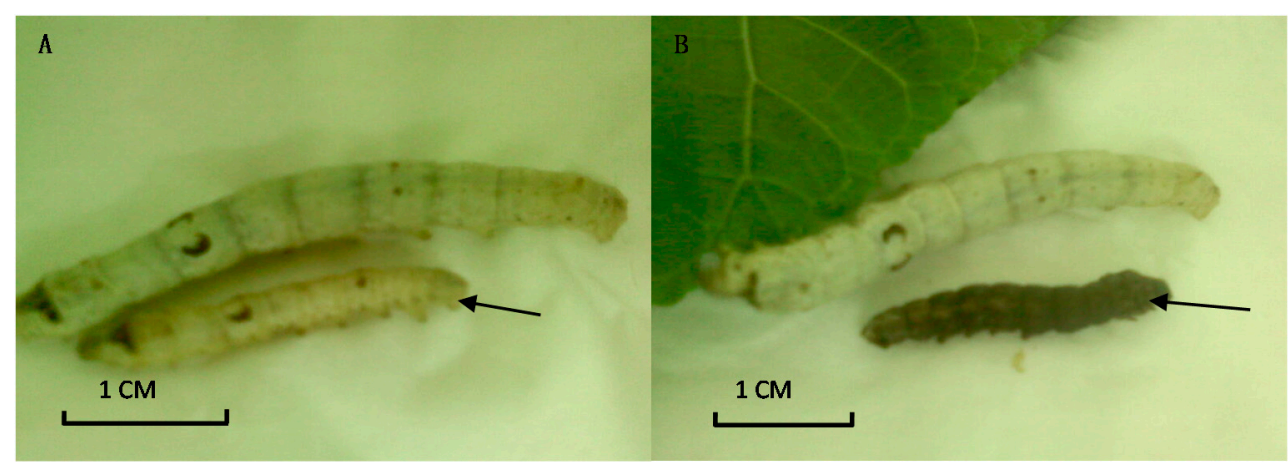

\section{Experimental Section}

\subsection{Insects}

The $B$. mori (strain: 1079) were maintained using mulberry leaves under the condition of $80 \%$ relative humidity and $25{ }^{\circ} \mathrm{C}$. For gene expression analysis, egg, larvae at the first day of each instar, pupa and adult $B$. mori were collected. The tissues of head, ovary, testis, Malpighian tubules and silk glands were 
dissected from the 5th instar larvae. All samples were frozen using liquid nitrogen and then kept at $-70{ }^{\circ} \mathrm{C}$ for RNA purification. All experiments were done in triplicates.

\subsection{RNA Purification and cDNA Synthesis}

The samples were frozen with liquid nitrogen and homogenized in a tissue grinder. Then, $1 \mathrm{~mL}$ TRIzol reagent (Invitrogen, Carlsbad, CA, USA) was added. Total RNA was isolated following the manufacturer's instructions. DNA was removed from total RNA by DNase I treatment following the protocol (Takara, Otsu, Japan). RNA integrity was checked on a 1.0\% agarose gel and visualized by ethidium bromide staining. The concentration and quality of RNA were measured with Nano drop ND-1000 (Thermo scientific, Wilmington, DE, USA). The first strand of cDNA template was synthesized from $1 \mu \mathrm{g}$ total RNA using M-MLV reverse transcriptase (Takara, Otsu, Japan) and Oligo (dT18) as the anchor primers. The reaction mixture was incubated at $70{ }^{\circ} \mathrm{C}$ for $10 \mathrm{~min}$ followed by $42{ }^{\circ} \mathrm{C}$ for $1 \mathrm{~h}$ and $70^{\circ} \mathrm{C}$ for $15 \mathrm{~min}$.

\subsection{Quantitative Real-Time PCR}

The quantitative real-time PCR (qPCR) reactions were carried out with SYBR Premix Ex Taq ${ }^{\mathrm{TM}}$ (Takara, Otsu, Japan) following the manufacturer's protocol using an ABI Prism 7300 (Applied Biosystems, Foster City, CA, USA). The reaction mixture contained $2 \mu \mathrm{L}$ cDNA template. The final reaction volume was $20 \mu \mathrm{L}$. The PCR reactions included an initial step of $95^{\circ} \mathrm{C}$ for $30 \mathrm{~s}$, followed by 40 cycles of $95{ }^{\circ} \mathrm{C}$ for $5 \mathrm{~s}$ and then annealed at $60{ }^{\circ} \mathrm{C}$ for $31 \mathrm{~s}$, one cycle of $95{ }^{\circ} \mathrm{C}$ for $15 \mathrm{~s}, 60{ }^{\circ} \mathrm{C}$ for $60 \mathrm{~s}$ and $95^{\circ} \mathrm{C}$ for $15 \mathrm{~s}$. The primers were designed with Beacon designer 7.0 (PREMIER Biosoft, Palo Alto, CA, USA) (Table 2). Amplification efficiencies were determined by template dilution. The qPCR specificity was monitored with melting curve analysis using the SDS software (2.2.1, Applied Biosystems, Foster City, CA, USA) and gel electrophoresis. The housekeeping gene RP49 was used as the reference genes for data normalization. The relative abundance was calculated using $2^{-\Delta \Delta C t}$ method [44]. The data were analyzed using the SPSS software (v19, SPSS, Chicago, IL, USA) $(p<0.05)$. All experiments were repeated in triplicates.

Table 2. The silkworm orthologs of human genes associated with diabetes mellitus.

\begin{tabular}{lll}
\hline Genes & Primer Names & Sequences \\
\hline \multirow{2}{*}{ Bm-INSR } & INSR-qRT-up & 5'-GGTCCGAGATGACTTGTATGG-3' \\
& INSR-qRT-down & 5'-GCTGCTGTTGAATGTCTTATAGG-3' \\
\hline \multirow{2}{*}{ Ribosomal } & rp49-qRT-up & 5'-AGGCATCAATCGGATCGCTATG-3' \\
protein 49 & rp49-qRT-down & 5'-TTGTGAACTAGGACCTTACGGAATC-3' \\
\multirow{2}{*}{ Bm-INSR } & INSR-dsRNA-up * & 5'-GGATCCTAATACGACTCACTATAGGAGACGCTATCATTGTTGGA-3' \\
& INSR-dsRNA-down & 5'-GGATCCTAATACGACTCACTATAGGTGCGGTTCACTTTACGA-3' \\
\multirow{2}{*}{ GFP } & GFP-dsRNA-up & 5'-GGATCCTAATACGACTCACTATAGGAAGTTCAGCGTGTCCG-3' \\
& GFP-dsRNA-down & 5'-GGATCCTAATACGACTCACTATAGGCACCTTGATGCCGTTC-3' \\
\hline \multicolumn{2}{c}{$*$ The sequences in box are the T7 promoter sequence for synthesis of dsRNA. }
\end{tabular}




\subsection{Double Strand RNA Synthesis}

Double strand RNA (dsRNA) was synthesized using T7 RiboMAX kit (Promega, Madison, WI, USA) following the manufacturer's protocol. The primers for amplifying dsRNA were designed according to the sequence of Bm-INSR (BGIBMGA003654) with primer premier 5.0 (Table 2). The PCR products were confirmed by sequencing. The dsRNA of GFP genes were used as the negative control.

\subsection{DsRNA Injection and Phenotype Observation}

For RNAi of $B m$-INSR gene, $1 \mu \mathrm{L}$ dsRNA $\left(5000 \mathrm{ng} \cdot \mu \mathrm{L}^{-1}\right)$ were injected into the $3 \mathrm{rd}$ instar larvae using micro-needles. Each group contained 50 insects. At $24 \mathrm{~h}$ after dsRNA injection, ten insects were selected from each group to measure body length and weight. Since then, phenotype observations were carried out every day until pupation. At 48, 96 and $124 \mathrm{~h}$ after dsRNA injection, six insects were randomly selected from each group to examine the abundance of Bm-INSR gene.

\subsection{Bioinformatics Analysis}

The accession numbers of human disease associated genes were downloaded from the database of Online Mendelian Inheritance in Man (OMIM) [37]. The protein sequences were obtained from the GenBank. The B. mori gene sequences were downloaded from the SilkDB database [45]. The orthologs were determined using bidirectional best hits (BBHs) of BLAST [46], which is a widely used method for identifying orthologs. The $E$-value cut-off is 0.00001 . The results were confirmed by searching the OrthoDB [47]. The domain analysis of INSR genes was conducted by CD-searching. Gene ontology analysis was conducted using the software Blast2go [48]. Pathway analysis was conducted by BLASTP against the KEGG database.

\section{Conclusions}

Animal models have been widely used in studying molecular mechanisms of human diseases and drug screens. Among these animal models, insects have received increasing attention because of short life cycles and convenience of rearing. Many successful experiments have been carried out in D. melanogaster. The fruitfly was used to model human diseases such as Alzheimer's disease [49], Parkinson disease [50], learning and memory [51], and insulin resistance [52,53]. The diamondback moth, Plutella xylostella, was used to study excess fat storage under different nutritional environments [54].

However, only several well-known insects were considered for developing disease model. There are many more economical or agricultural important insects with genome sequences available. $B$. mori has been domesticated for thousands of years [55]. The genome of B. mori was published almost ten years ago [23]. The silkworm has been used in traditional Chinese medicine for a very long time [56,57]. B. $m o r i$ is also an efficient bioreactor to produce vaccine and drug-related proteins $[29,58,59]$. In this work, bioinformatics analysis demonstrated that more than $5000 \mathrm{~B}$. mori genes were the orthologs of human disease associated. These B. mori genes are rich in metabolic pathways, suggesting that $B$. mori might be a suitable animal model for metabolic syndrome and associated complications.

In the ancient medical texts of traditional Chinese medicine, diabetes-related symptoms were called as "Xiaoke" disease. Reference mining of medical texts such as Huang Di Nei Jing, the Yellow 
Emperor's Inner Classic, and approximately 1200 recipes and 150 herbs for diabetes demonstrated that B. mori and its moths were widely used to treat "Xiaoke" patients in ancient China [60-63]. Here, we chose Bm-INSR gene as an example to study the possibility of using B. mori as diabetes model. Expression analysis showed that Bm-INSR was highly expressed at the 3rd and the 4th instar larvae, which was consistent with eating habit of the B. mori. RNAi of Bm-INSR resulted in high percentages of black body color, which is similar in diabetes patients.

Bombyxin in $B$. mori is the first insulin-like peptide discovered in insects, which shares $56 \%$ amino acid similarity with human insulin [64]. The insulin/insulin-like signaling pathways are highly conserved between vertebrate and invertebrate, which is an important basis of using the B. mori to model diabetes [65]. The insulin receptor is associated with diabetes in human, however, there is not enough data from insects; this is worthy of further investigation. In summary, we presented experimental evidence that $B$. mori is a suitable animal model for diabetes mellitus. The "diabetes silkworm" can be easily obtained by RNA interference.

\section{Supplementary Materials}

Supplementary materials can be found at http://www.mdpi.com/1422-0067/15/10/18102/s1.

\section{Acknowledgments}

We thank Muwang Li from the Sericulture Research Institute, CAAS, China for providing the silkworm. This work was in partial supported by National Science Foundation of China (31171843, 31301691 and 31071687), Jiangsu Science Foundation for Distinguished Young Scholars (BK2012028).

\section{Author Contributions}

Fei Li conceived and designed the experiments; Zan Zhang conducted bioinformatics analysis; Xiaolu Teng performed the molecular experiments; Fei Li, Zan Zhang, Xiaolu Teng and Maohua Chen wrote the paper.

\section{Conflicts of Interest}

The authors declare no conflict of interest.

\section{References}

1. Espinosa-Oliva, A.M.; de Pablos, R.M.; Herrera, A.J. Intracranial injection of lps in rat as animal model of neuroinflammation. Methods Mol. Biol. 2013, 1041, 295-305.

2. Harrison, C. Animal models: Capturing ad features in a novel rat model. Nat. Rev. Drug Discov. 2013, doi:10.1038/nrd4041.

3. Sasase, T.; Ohta, T.; Masuyama, T.; Yokoi, N.; Kakehashi, A.; Shinohara, M. The spontaneously diabetic torii rat: An animal model of nonobese type 2 diabetes with severe diabetic complications. J. Diabetes Res. 2013, 2013, 976209.

4. Kreiss, D.S.; Coffman, C.F.; Fiacco, N.R.; Granger, J.C.; Helton, B.M.; Jackson, J.C.; Kim, L.V.; Mistry, R.S.; Mizer, T.M.; Palmer, L.V.; et al. Ritualistic chewing behavior induced by mCPP in 
the rat is an animal model of obsessive compulsive disorder. Pharmacol. Biochem. Behav. 2013, 104, 119-124.

5. Ding, S.; Wu, X.; Li, G.; Han, M.; Zhuang, Y.; Xu, T. Efficient transposition of the piggyBac (Pb) transposon in mammalian cells and mice. Cell 2005, 122, 473-483.

6. Marconato, L.; Gelain, M.E.; Comazzi, S. The dog as a possible animal model for human non-Hodgkin lymphoma: A review. Hematol. Oncol. 2013, 31, 1-9.

7. Steffan, J.S.; Bodai, L.; Pallos, J.; Poelman, M.; McCampbell, A.; Apostol, B.L.; Kazantsev, A.; Schmidt, E.; Zhu, Y.Z.; Greenwald, M.; et al. Histone deacetylase inhibitors arrest polyglutamine-dependent neurodegeneration in Drosophila. Nature 2001, 413, 739-743.

8. Bilen, J.; Bonini, N.M. Drosophila as a model for human neurodegenerative disease. Annu. Rev. Genet. 2005, 39, 153-171.

9. Jung, S.H.; Evans, C.J.; Uemura, C.; Banerjee, U. The Drosophila lymph gland as a developmental model of hematopoiesis. Development 2005, 132, 2521-2533.

10. Marsh, J.L.; Pallos, J.; Thompson, L.M. Fly models of Huntington's disease. Hum. Mol. Genet. 2003, 12, R187-R193.

11. Xu, Y.; Condell, M.; Plesken, H.; Edelman-Novemsky, I.; Ma, J.; Ren, M.; Schlame, M. A Drosophila model of barth syndrome. Proc. Natl. Acad. Sci. USA 2006, 103, 11584-11588.

12. Whitworth, A.J.; Theodore, D.A.; Greene, J.C.; Benes, H.; Wes, P.D.; Pallanck, L.J. Increased glutathione $S$-transferase activity rescues dopaminergic neuron loss in a Drosophila model of Parkinson's disease. Proc. Natl. Acad. Sci. USA 2005, 102, 8024-8029.

13. Crowther, D.C.; Kinghorn, K.J.; Miranda, E.; Page, R.; Curry, J.A.; Duthie, F.A.; Gubb, D.C.; Lomas, D.A. Intraneuronal A $\beta$, non-amyloid aggregates and neurodegeneration in a Drosophila model of Alzheimer's disease. Neuroscience 2005, 132, 123-135.

14. Vidal, M.; Cagan, R.L. Drosophila models for cancer research. Curr. Opin. Genet. Dev. 2006, 16, $10-16$.

15. Bier, E.; Bodmer, R. Drosophila, an emerging model for cardiac disease. Gene 2004, 342, 1-11.

16. Reiter, L.T.; Potocki, L.; Chien, S.; Gribskov, M.; Bier, E. A systematic analysis of human disease-associated gene sequences in Drosophila melanogaster. Genome Res. 2001, 11, 1114-1125.

17. Wolf, M.J.; Amrein, H.; Izatt, J.A.; Choma, M.A.; Reedy, M.C.; Rockman, H.A. Drosophila as a model for the identification of genes causing adult human heart disease. Proc. Natl. Acad. Sci. USA 2006, 103, 1394-1399.

18. Read, R.D.; Goodfellow, P.J.; Mardis, E.R.; Novak, N.; Armstrong, J.R.; Cagan, R.L. A Drosophila model of multiple endocrine neoplasia type 2. Genetics 2005, 171, 1057-1081.

19. Mehta, V.; Peebles, D.; David, A.L. Animal models for prenatal gene therapy: Choosing the right model. Methods Mol. Biol. 2012, 891, 183-200.

20. Little, C.B.; Zaki, S. What constitutes an "animal model of osteoarthritis"-The need for consensus? Osteoarthr. Cartil. 2012, 20, 261-267.

21. Salkovic-Petrisic, M.; Knezovic, A.; Hoyer, S.; Riederer, P. What have we learned from the streptozotocin-induced animal model of sporadic Alzheimer's disease, about the therapeutic strategies in Alzheimer's research. J. Neural Transm. 2013, 120, 233-252. 
22. Xia, Q.; Zhou, Z.; Lu, C.; Cheng, D.; Dai, F.; Li, B.; Zhao, P.; Zha, X.; Cheng, T.; Chai, C.; et al. A draft sequence for the genome of the domesticated silkworm (Bombyx mori). Science 2004, 306, 1937-1940.

23. Mita, K.; Kasahara, M.; Sasaki, S.; Nagayasu, Y.; Yamada, T.; Kanamori, H.; Namiki, N.; Kitagawa, M.; Yamashita, H.; Yasukochi, Y.; et al. The genome sequence of silkworm, Bombyx mori. DNA Res. 2004, 11, 27-35.

24. Deng, D.; Xu, H.; Wang, F.; Duan, X.; Ma, S.; Xiang, Z.; Xia, Q. The promoter of Bmlp3 gene can direct fat body-specific expression in the transgenic silkworm, Bombyx mori. Transgenic Res. 2013, $22,1055-1063$

25. Jiang, L.; Zhao, P.; Wang, G.; Cheng, T.; Yang, Q.; Jin, S.; Lin, P.; Xiao, Y.; Sun, Q.; Xia, Q. Comparison of factors that may affect the inhibitory efficacy of transgenic RNAi targeting of baculoviral genes in silkworm, Bombyx mori. Antivir. Res. 2013, 97, 255-263.

26. Sato, M.; Kojima, K.; Sakuma, C.; Murakami, M.; Aratani, E.; Takenouchi, T.; Tamada, Y.; Kitani, H. Production of scFv-conjugated affinity silk powder by transgenic silkworm technology. PLoS One 2012, 7, e34632.

27. Gong, Z.H.; Jin, H.Q.; Jin, Y.F.; Zhang, Y.Z. Expression of cholera toxin B subunit and assembly as functional oligomers in silkworm. J. Biochem. Mol. Biol. 2005, 38, 717-724.

28. Du, D.; Kato, T.; Nabi, A.H.; Suzuki, F.; Park, E.Y. Expression of functional human (pro)renin receptor in silkworm (Bombyx mori) larvae using BmMNPV bacmid. Biotechnol. Appl. Biochem. 2008, 49, 195-202.

29. Liu, Y.; Chen, Y.; Chen, J.; Zhang, W.; Sheng, Q.; Chen, J.; Yu, W.; Nie, Z.; Zhang, Y.; Wu, W.; et al. A shark liver gene-derived active peptide expressed in the silkworm, Bombyx mori: Preliminary studies for oral administration of the recombinant protein. Mar. Drugs 2013, 11, $1492-1505$.

30. Tabunoki, H.; Ono, H.; Ode, H.; Ishikawa, K.; Kawana, N.; Banno, Y.; Shimada, T.; Nakamura, Y.; Yamamoto, K.; Satoh, J.; et al. Identification of key uric acid synthesis pathway in a unique mutant silkworm Bombyx mori model of Parkinson's disease. PLoS One 2013, 8, e69130.

31. Meng, Y.; Katsuma, S.; Daimon, T.; Banno, Y.; Uchino, K.; Sezutsu, H.; Tamura, T.; Mita, K.; Shimada, T. The silkworm mutant lemon (lemon lethal) is a potential insect model for human sepiapterin reductase deficiency. J. Biol. Chem. 2009, 284, 11698-11705.

32. Matsumoto, Y.; Sumiya, E.; Sugita, T.; Sekimizu, K. An invertebrate hyperglycemic model for the identification of anti-diabetic drugs. PLoS One 2011, 6, e18292.

33. Feng, F.; Chen, L.; Lian, C.; Xia, H.; Zhou, Y.; Yao, Q.; Chen, K. Comparative proteomic analysis reveals the suppressive effects of dietary high glucose on the midgut growth of silkworm. J. Proteomics 2014, 108, 124-132.

34. Satake, S.; Masumura, M.; Ishizaki, H.; Nagata, K.; Kataoka, H.; Suzuki, A.; Mizoguchi, A. Bombyxin, an insulin-related peptide of insects, reduces the major storage carbohydrates in the silkworm Bombyx mori. Comp. Biochem. Physiol. 1997, 118, 349-357.

35. Iwami, M. Bombyxin: An insect brain peptide that belongs to the insulin family. Zool. Sci. 2000, 17, 1035-1044. 
36. Masumura, M.; Satake, S.; Saegusa, H.; Mizoguchi, A. Glucose stimulates the release of bombyxin, an insulin-related peptide of the silkworm Bombyx mori. Gen. Comp. Endocrinol. 2000, 118, 393-399.

37. Online Mendelian Inheritance in Man. Available online: http://omia.angis.org.au/home/ (accessed on 10 November 2011).

38. Huang, H.; Winter, E.E.; Wang, H.; Weinstock, K.G.; Xing, H.; Goodstadt, L.; Stenson, P.D.; Cooper, D.N.; Smith, D.; Alba, M.M.; et al. Evolutionary conservation and selection of human disease gene orthologs in the rat and mouse genomes. Genome Biol. 2004, 5, R47.

39. Pautsch, A.; Zoephel, A.; Ahorn, H.; Spevak, W.; Hauptmann, R.; Nar, H. Crystal structure of bisphosphorylated IGF-1 receptor kinase: Insight into domain movements upon kinase activation. Structure 2001, 9, 955-965.

40. Bansilal, S.; Farkouh, M.E.; Fuster, V. Role of insulin resistance and hyperglycemia in the development of atherosclerosis. Am. J. Cardiol. 2007, 99, 6B-14B.

41. Kikuta, S.; Hagiwara-Komoda, Y.; Noda, H.; Kikawada, T. A novel member of the trehalose transporter family functions as an $\mathrm{H}^{+}$-dependent trehalose transporter in the reabsorption of trehalose in malpighian tubules. Front. Physiol. 2012, 3, 290.

42. Schertzer, J.D.; Tamrakar, A.K.; Magalhaes, J.G.; Pereira, S.; Bilan, P.J.; Fullerton, M.D.; Liu, Z.; Steinberg, G.R.; Giacca, A.; Philpott, D.J.; et al. Nod1 activators link innate immunity to insulin resistance. Diabetes 2011, 60, 2206-2215.

43. Steinberg, G.R. Inflammation in obesity is the common link between defects in fatty acid metabolism and insulin resistance. Cell Cycle 2007, 6, 888-894.

44. Pfaffl, M.W. A new mathematical model for relative quantification in real-time RT-PCR. Nucleic Acids Res. 2001, 29, e45.

45. Silkdb. Available online: Ftp://silkdb.org/ (accessed on 12 November 2011).

46. Altschul, S.F.; Gish, W.; Miller, W.; Myers, E.W.; Lipman, D.J. Basic local alignment search tool. J. Mol. Biol. 1990, 215, 403-410.

47. Waterhouse, R.M.; Tegenfeldt, F.; Li, J.; Zdobnov, E.M.; Kriventseva, E.V. Orthodb: A hierarchical catalog of animal, fungal and bacterial orthologs. Nucleic Acids Res. 2013, 41, D358-D365.

48. Gotz, S.; Garcia-Gomez, J.M.; Terol, J.; Williams, T.D.; Nagaraj, S.H.; Nueda, M.J.; Robles, M.; Talon, M.; Dopazo, J.; Conesa, A. High-throughput functional annotation and data mining with the Blast2GO suite. Nucleic Acids Res. 2008, 36, 3420-3435.

49. Costa, R.; Speretta, E.; Crowther, D.C.; Cardoso, I. Testing the therapeutic potential of doxycycline in a Drosophila melanogaster model of Alzheimer disease. J. Biol. Chem. 2011, 286, 41647-41655.

50. Kim, K.; Kim, S.H.; Kim, J.; Kim, H.; Yim, J. Glutathione $S$-transferase $\omega 1$ activity is sufficient to suppress neurodegeneration in a Drosophila model of Parkinson disease. J. Biol. Chem. 2012, 287, 6628-6641.

51. Qin, H.; Cressy, M.; Li, W.; Coravos, J.S.; Izzi, S.A.; Dubnau, J. $\gamma$ Neurons mediate dopaminergic input during aversive olfactory memory formation in Drosophila. Curr. Biol. 2012, 22, 608-614.

52. Morris, S.N.; Coogan, C.; Chamseddin, K.; Fernandez-Kim, S.O.; Kolli, S.; Keller, J.N.; Bauer, J.H. Development of diet-induced insulin resistance in adult Drosophila melanogaster. Biochim. Biophys. Acta 2012, 1822, 1230-1237. 
53. Pasco, M.Y.; Leopold, P. High sugar-induced insulin resistance in Drosophila relies on the lipocalin Neural Lazarillo. PLoS One 2012, 7, e36583.

54. Warbrick-Smith, J.; Behmer, S.T.; Lee, K.P.; Raubenheimer, D.; Simpson, S.J. Evolving resistance to obesity in an insect. Proc. Natl. Acad. Sci. USA 2006, 103, 14045-14049.

55. Dai, L.S.; Zhu, B.J.; Liu, Q.N.; Wei, G.Q.; Liu, C.L. Characterization of the complete mitochondrial genome of Bombyx mori strain h9 (Lepidoptera: Bombycidae). Gene 2013, 519, 326-334.

56. Kikuchi, H.; Takahashi, N.; Oshima, Y. Novel aromatics bearing 4-O-methylglucose unit isolated from the oriental crude drug Bombyx Batryticatus. Tetrahedron Lett. 2004, 45, 367-370.

57. Hu, K.; Wang, Q.M.; Hu, P.Q. The male silkworm moth (Antheraea pernyi) is a key ingredient in $\mathrm{Hu}-\mathrm{Bao}$ and Sheng-Bao for specific prolongation of the life-span of the male fruit fly (Drosophila melanogaster). Am. J. Chin. Med. 2002, 30, 263-270.

58. Gong, Z.; Jin, Y.; Zhang, Y. Oral administration of a cholera toxin B subunit-insulin fusion protein produced in silkworm protects against autoimmune diabetes. J. Biotechnol. 2005, 119, 93-105.

59. Cong, L.; Cao, G.; Renyu, X.; Zhonghua, P.; Xiaojian, Z.; Zhou, W.; Gong, C. Reducing blood glucose level in tidm mice by orally administering the silk glands of transgenic $h I G F$-I silkworms. Biochem. Biophys. Res. Commun. 2011, 410, 721-725.

60. Zhao, H.L.; Tong, P.C.; Chan, J.C. Traditional Chinese medicine in the treatment of diabetes. Nestle Nutr. Workshop Ser. Clin. Perform. Programme 2006, 11, 15-25; discussion 25-19.

61. Zhang, T.T.; Jiang, J.G. Active ingredients of traditional Chinese medicine in the treatment of diabetes and diabetic complications. Expert Opin. Investig. Drugs 2012, 21, 1625-1642.

62. Tong, X.L.; Dong, L.; Chen, L.; Zhen, Z. Treatment of diabetes using traditional Chinese medicine: Past, present and future. Am. J. Chin. Med. 2012, 40, 877-886.

63. Kasuli, E.G. Are alternative supplements effective treatment for diabetes mellitus? Nutr. Clin. Pract. 2011, 26, 352-355.

64. Wu, Q.; Brown, M.R. Signaling and function of insulin-like peptides in insects. Annu. Rev. Entomol. 2006, 51, 1-24.

65. Dou, T.; Ji, C.; Gu, S.; Xu, J.; Xu, J.; Ying, K.; Xie, Y.; Mao, Y. Co-evolutionary analysis of insulin/insulin like growth factor 1 signal pathway in vertebrate species. Front. Biosci. 2006, 11, 380-388.

(C) 2014 by the authors; licensee MDPI, Basel, Switzerland. This article is an open access article distributed under the terms and conditions of the Creative Commons Attribution license (http://creativecommons.org/licenses/by/4.0/). 